\title{
La problemática acerca de la conclusión del silogismo de la primera figura
}

THE PROBLEM ON THE CONCLUSION OF THE SYLLOGISM IN THE FIRST FIGURE

Dr. Miguel López-Astorga (milopez@utalca.cl) Instituto de Estudios Humanísticos Juan Ignacio Molina, Universidad de Talca (Talca, Chile)

\begin{abstract}
Espino and Santamaria experimentally demonstrate that, if the syllogisms in the first figure are not presented as is the usual practice, that is, separating their two premises into two different lines, but linking the two premises into one line by means of a full stop, individuals tend to draw from such premises conclusions in which the subject and the predicate are not in the customary order, but rotated. In their view, this finding is consistent with the assumptions of the mental models theory. Nevertheless, in this paper, I will show that what this fact really depends on the approach that is adopted, since a framework based on formal logic also has resources to explain it, and hence can lead us to assume theses different from those proposed by Espino and Santamaria.
\end{abstract}

Key words: first figure, formal logic, inferential activity, mental models, syllogism.

\section{Resumen}

Espino y Santamaría demuestran experimentalmente que, si los silogismos de la primera figura no son planteados como se hace tradicionalmente, esto es, separando sus dos premisas en dos líneas diferentes, sino en una sola línea uniendo las dos premisas mediante un punto seguido, los individuos tienden a deducir a partir de tales premisas conclusiones en las que el sujeto y el predicado no se encuentran en el orden habitual, sino invertidos. Desde su punto de vista, este hallazgo es consistente con los supuestos de la teoría de los modelos mentales. No obstante, en este trabajo, vamos a mostrar que lo que tal hecho significa verdaderamente depende del enfoque que se adopte, ya que un marco basado en la lógica formal también cuenta con los recursos para explicarlo y, por tanto, puede conducirnos a asumir tesis diferentes a las propuestas por Espino y Santamaría.

Palabras clave: actividad inferencial, lógica formal, modelos mentales, primera figura, silogismo. 


\section{Introducción}

Espino y Santamaría (2013) presentan un experimento que conduce a hallazgos muy interesantes y de gran relevancia. Entre ellos, el más importante, a nuestro juicio, es que los individuos pueden alterar el orden de los términos de las conclusiones de los silogismos de la primera figura si sus premisas no son expresadas en dos renglones distintos, sino en un mismo renglón y vinculadas por medio de un punto seguido. Como sabemos, las premisas del silogismo de la primera figura se atienen a esta estructura:

\section{B-A}

C-B

Y, como también es conocido, la conclusión que, desde el punto de vista aristotélico, corresponde a esta figura tiene la siguiente forma:

\section{C-A}

De hecho, como nos recuerdan Espino y Santamaría, la literatura nos confirma que, cuando se le presenta a un grupo de participantes las dos premisas del silogismo de esta figura y se les solicita que extraigan ellos la conclusión, la mayoría tiende a responder con conclusiones acordes con el esquema C-A. Esto puede comprobarse, por ejemplo, en trabajos como el de Johnson-Laird y Steedman (1978) que por razones de argumentación no se vuelven a describir aquí.

No obstante, como decimos, Espino y Santamaría descubren que, si las dos premisas se exponen al participante en una misma línea y unidas por un punto seguido (esto es, siguiendo la estructura B-A. C-B), estos tienden a inferir conclusiones con los dos términos (sujeto y predicado) invertidos (esto es, conclusiones con la forma $\mathrm{A}-\mathrm{C}$ ). Este hecho, nos comentan, es absolutamente consistente con las tesis sobre esta temática que se han propuesto desde la teoría de los modelos mentales (en adelante, vamos a referirnos a esta teoría mediante las siglas MMT, correspondientes a la expresión en inglés Mental Models Theory). MMT es una teoría que, sin duda, ha tenido mucho éxito en los últimos tiempos y que ha demostrado ser indiscutiblemente eficaz para explicar diversas problemáticas. Es muy extensa la producción que se puede encontrar con respecto a ella y, por esta razón, sólo remitimos a los trabajos de Philip N. Johnson-Laird y sus colaboradores.

Que los resultados obtenidos por Espino y Santamaría sean coherentes con MMT es claramente una evidencia más a favor de esta teoría y en este trabajo no vamos a defender lo contrario. Empero, sí vamos a argumentar que tales resultados pueden ser igualmente interpretados desde un enfoque lógico-formal y que el cálculo de deducción natural tipo Gentzen permite dos procesos de derivación a partir de las premisas correspondientes al silogismo de la primera figura, uno de los cuales se puede vincular con C-A y el otro con A-C.

Esto no significa, sin embargo, que adoptemos un marco con lineamientos semejantes a los que se pueden encontrar en la producción de autores como David P. O'Brien o Lance Rips, por nombrar solo algunas propuestas, según los cuales el razonamiento humano se atiene básicamente a prescripciones sintácticas. Simplemente, pretendemos evidenciar que existen ciertas dificultades metodológicas que revisten a esta problemática y que hacen referencia a que los mismos datos experimentales pueden ser considerados como compatibles con supuestos distintos o, incluso, opuestos. Y es que, como vamos a comprobar más abajo, un enfoque basado en el cálculo de predicados de primer orden (en lo que sigue, vamos a utilizar 
las siglas FOPC, procedentes de la expresión en inglés First-Order Predicate Calculus, para aludir a este cálculo) parece, al menos, contar con los recursos necesarios para elaborar una explicación alternativa a la que plantea MMT para la temática que estamos analizando.

Entendemos, sin embargo, que, antes de proponer argumentos en este sentido, puede ser oportuno describir con mayor detalle en qué consistió exactamente el experimento de Espino y Santamaría y mostrar por qué sus resultados confirmaron las predicciones que ellos derivaron de los supuestos de MMT. A esto dedicamos las siguientes páginas.

\section{Las dos formas posibles de la conclusión del silogismo de la primera figura}

En realidad, el experimento de Espino y Santamaría es mucho más amplio, pues trabajan con las cuatro figuras aristotélicas -no sólo con la primera- y obtienen resultados relevantes para todas ellas. No obstante, en estas páginas, nos interesan fundamentalmente los relativos a la primera figura.

Quizás, para explicar con mayor claridad las características generales de su labor experimental en lo relativo a la primera figura, puede ser oportuno recurrir a un ejemplo temático que ellos mismos incluyen en su trabajo. Tal ejemplo es el siguiente:

P1L (presentación en una línea):

"All of the British are Artists. Some of the Teachers are British" (Espino y Santamaría 2013:322).

P2L (presentación en dos líneas):

"All of the British are Artists.

Some of the Teachers are British"

Las traducciones al español de estas dos presentaciones, que son con las que vamos a trabajar en lo que sigue, son estas:

P1L:

Todos los británicos son artistas. Algunos profesores son británicos.

P2L:

Todos los británicos son artistas.

Algunos profesores son británicos. 
El gran hallazgo de Espino y Santamaría, por lo que a este asunto se refiere, es, como hemos apuntado, que el patrón de respuestas no fue exactamente el mismo con estas dos presentaciones del problema. Cuando utilizaron la presentación habitual (esto es, P2L) y preguntaron a sus participantes qué se seguía de esa información, estos respondieron mayoritariamente que algunos profesores son artistas (esto es, respondieron mayoritariamente de acuerdo con la estructura C-A). No obstante, cuando, preguntando lo mismo, plantearon la tarea con el formato P1L, aumentó significativamente el número de participantes que contestaron que algunos artistas son profesores (esto es, que contestaron en correspondencia con la estructura $\mathrm{A}-\mathrm{C})$.

Espino y Santamaría reconocen que se han propuesto diversas teorías con respecto a la temática de los silogismos y sus conclusiones, pero, desde su punto de vista, ninguna de ellas, a excepción de MMT, posee los recursos suficientes o la maquinaria necesaria para explicar sus resultados. Entre tales enfoques, citan, por ejemplo, los presentados en el artículo de Chater y Oaksford (The probability heuristics model of syllogistic reasoning) de 1999 en la revista Cognitive Psychology, en el de Ford (Two models of mental representation and problem solution in syllogistic reasoning) de 1995 en la revista Cognition y en el de Oberauer y Wilhelm (Effects of directionality in deductive reasoning: I. The comprehension of single relational premises) de 2000 en la revista Journal of Experimental Psychology: Learning, Memory and Cognition, y, efectivamente, sus argumentos revelan que, desde marcos como estos, difícilmente se puede explicar por qué P2L conduce a respuestas del tipo C-A y P1L suele ir acompañada de respuestas del tipo A-C. MMT, tal y como Espino y Santamaría señalan, puede, sin duda, ofrecer una explicación al respecto. No obstante, como hemos apuntado, la lógica formal también cuenta con ciertas posibilidades en este sentido que pueden llevarnos a una concepción diferente de los procesos cognitivos humanos. Parece oportuno, empero, antes de exponer las potencialidades de la lógica formal en lo relativo a esta problemática, describir los supuestos de MMT que permiten comprender los resultados de Espino y Santamaría.

\section{MMT y las conclusiones de los silogismos}

Espino y Santamaría apoyan su argumentación en trabajos como el libro de Johnson-Laird (How we reason) de 2006, el artículo de Johnson-Laird y Bara (Syllogistic inference) de 1984 en la revista Cognition y el texto de Johnson-Laird y Byrne (Deduction) de 1991 y adoptan el principio FIFO (siglas procedentes de la expresión en inglés First In, First Out), según el cual la primera pieza de información que forma parte del modelo integrado de las premisas de un silogismo se convierte en el sujeto de la conclusión. Esto significa, como nos comentan Espino y Santamaría, que, cuando los términos medios de un silogismo se dan en secuencia, como sucede, por ejemplo, en el caso de la cuarta figura (A-B / B-C), el primer término del modelo es $\mathrm{A}$ y, en virtud del principio FIFO, la conclusión más frecuente es $\mathrm{A}-\mathrm{C}$.

Desde esta óptica, el problema surge cuando los términos medios no se presentan en secuencia, que es precisamente lo que sucede en la primera figura aristotélica. Basándose en el artículo de Johnson-Laird y Bara mencionado, Espino y Santamaría afirman que, cuando este es el caso, el individuo cuenta con dos procedimientos posibles para construir su modelo. El primero de ellos consiste en rotar los términos de las premisas ( $\mathrm{B}-\mathrm{A}$ y $\mathrm{C}-\mathrm{B}$ ) en la memoria de trabajo, lo que permite conectar los dos términos medios, dando lugar a una estructura como A-B / B-C. Cuando esto sucede, FIFO provoca que A se transforme en el sujeto de la conclusión. 
El segundo procedimiento apunta a la acción de invertir el orden de los modelos de las premisas, resultando una estructura final con la forma C-B / B-A. En estas situaciones, obviamente, FIFO conduce a que el sujeto de la conclusión sea $C$.

Así, reconociendo que no está claro cuál es el motivo por el que los individuos se decantan en unas ocasiones por uno de estos procedimientos y en otras por el otro, Espino y Santamaría predijeron que sus participantes iban a preferir la primera estrategia ( $\mathrm{y}$, por tanto, a ofrecer respuestas con la estructura AC) ante tareas con silogismos con el formato P1L, y que, por el contrario, se iban a inclinar por la segunda estrategia ( $y$, por tanto, sus respuestas mayoritarias iban a tener la estructura $C-A$ ) ante tareas con silogismos con el formato P2L. No cabe duda, como señalamos más arriba, de que sus resultados confirmaron estas predicciones. Sin embargo, como también hemos indicado, si adoptamos un enfoque basado en la lógica formal, es posible notar ciertas circunstancias que pueden revelarnos un camino explicativo alternativo y mostrarnos que, si nos aproximamos a los resultados experimentales de Espino y Santamaría desde otra perspectiva, determinados fenómenos cognitivos pueden guiarnos hacia conclusiones diferentes. Presentamos nuestros argumentos en este sentido en el apartado siguiente.

\section{La lógica de predicados de primer orden y las conclusiones de los silogismos}

Para comprobar cómo la lógica formal cuenta también con recursos para enfrentar la problemática analizada por Espino y Santamaría, vamos a ceñirnos exclusivamente al ejemplo que ellos indican y al que hemos hecho referencia más arriba, esto es, al ejemplo de los británicos, los artistas y los profesores. Entendemos que una explicación basada en dicho ejemplo es suficiente porque, como se va a poder apreciar en líneas posteriores, es obvio cómo podría ser extrapolada a cualquier otro ejemplo con contenido temático.

Lo primero que precisamos para nuestra explicación es recurrir al cálculo apropiado para el razonamiento acerca de silogismos como el mencionado, el cual no es otro que FOPC. Para formalizar las premisas del ejemplo propuesto por Espino y Santamaría en términos de FOPC, necesitamos previamente establecer algunas equivalencias:

\section{B: 'ser británico' \\ A: 'ser artista' \\ P: 'ser profesor'}

De este modo, la primera premisa puede ser formalizada como $\forall x(B x \rightarrow A x)$. Si ' $\forall$ ' es el cuantificador universal $y$ ' $\rightarrow$ ' el condicional lógico, este enunciado se puede leer como 'Para todo $x$, si $x$ es británico, entonces $x$ es artista'. Por su parte, la segunda premisa puede ser formalizada como $\exists x\left(P x \wedge B^{\wedge x}\right)$. Si ' $\exists^{\prime}$ es el cuantificador existencial $y$ ' $\wedge$ ' la conjunción lógica, esta fórmula puede leerse como 'Existe un $x$ tal que $x$ es profesor y $x$ es británico'. A partir de aquí, es evidente cómo se puede derivar la conclusión por medio del cálculo tipo Gentzen: 
1) $\forall x(B x \rightarrow A x)$ (premisa)

2) $\exists x(P x \wedge B x)$ (premisa)

3) $\mathrm{Pa} \wedge \mathrm{Ba}$ (suposición)

4) $\mathrm{Ba} \rightarrow \mathrm{Aa}(\mathrm{ECU} 1)$

5) $\mathrm{Pa}(\mathrm{EC} 3)$

6) $\mathrm{Ba}(\mathrm{EC} \mathrm{3)}$

7) $\mathrm{Aa}(\mathrm{MP} 4,6)$

8) $\mathrm{Pa} \wedge \mathrm{Aa}(\mathrm{IC} 5,7)$

9) $\exists x(P x \wedge A x)(I C E 8)$

10) $\exists x(P x \wedge A x)(E C E 3-9)$

Con respecto a las abreviaturas entre paréntesis, ECU hace referencia a la regla de eliminación del cuantificador universal $(\forall x \mathrm{Px}+\mathrm{Pa}), \mathrm{EC}$ a la de eliminación de la conjunción $\left(\mathrm{p}^{\wedge} \mathrm{q}+\mathrm{p} / \mathrm{p}^{\wedge} \mathrm{q}+\mathrm{q}\right), \mathrm{MP}$ a la de Modus Ponens $(p \rightarrow q, p \vdash q)$, IC a la de introducción de la conjunción ( $p, q \vdash p \wedge q$ ), ICE a la de introducción del cuantificador existencial $(\mathrm{Pa} \vdash \exists x \mathrm{Px}$ ) y ECE a la de eliminación del cuantificador existencial (si $\mathrm{Pa} \vdash \mathrm{Z}$, entonces $\exists x \mathrm{Px}+\mathrm{Z}$, siempre que a no aparezca ni en $\exists x \mathrm{Px}$ ni en $\mathrm{Z}$ ni en ninguna suposición no cancelada).

Como se puede apreciar, esta derivación conduce a una conclusión con la forma C-A y, por tanto, podemos vincularla con P2L, ya que los participantes de Espino y Santamaría tendieron a ofrecer conclusiones con dicha forma ante este tipo de presentación. No obstante, el cálculo tipo Gentzen permite también una derivación con una conclusión con la forma A-C. Para ella, son necesarias exactamente las mismas reglas que hemos utilizado en la deducción anterior. Lo único que precisamos es modificar el orden de los pasos 5,6 y 7 de la siguiente manera:

1) $\forall x(B x \rightarrow A x)$ (premisa)

2) $\exists x(P x \wedge B x)$ (premisa)

3) $\mathrm{Pa} \wedge \mathrm{Ba}$ (suposición)

4) $\mathrm{Ba} \rightarrow \mathrm{Aa}(\mathrm{ECU} 1)$

5) $\mathrm{Ba}(\mathrm{EC} 3)$

6) $\mathrm{Aa}(\mathrm{MP} \mathrm{4,} \mathrm{5)}$

7) $\mathrm{Pa}(\mathrm{EC} 3)$ 
8) $\mathrm{Aa} \wedge \mathrm{Pa}(\mathrm{IC} 6,7)$

9) $\exists x\left(A x^{\wedge} P x\right)(I C E 8)$

10) $\exists x(A x \wedge P x)(E C E 3-9)$

La forma de la conclusión es en este caso A-C y, por ello, podemos decir que, de alguna manera, esta derivación corresponde a P1L, esto es, al tipo de presentación que condujo a los participantes de Espino y Santamaría a responder mayoritariamente que la conclusión se ajustaba a la estructura A-C.

Vemos, por consiguiente, que la lógica formal cuenta con los recursos necesarios para explicar la diferencia entre los patrones de respuesta asociados a P1L y P2L en el experimento planteado por Espino y Santamaría.

Naturalmente, quedan dos dudas que despejar: 1) cuál es el procedimiento exacto por el que las representaciones mentales de los individuos se transforman en fórmulas de FOPC y 2 ) en virtud de qué motivo o causa $\mathrm{P} 1 \mathrm{~L}$ se vincula con un orden determinado de los pasos 5,6 y 7 de la derivación y $\mathrm{P} 2 \mathrm{~L}$ con otro orden distinto de esos mismos pasos. No obstante, como hemos comprobado, se puede decir que en la lógica encontramos factores que pueden ayudar a proponer explicaciones para problemáticas como la que estamos considerando. No cabe duda de que cualquier explicación que pueda ofrecerse al respecto a partir de lo expuesto en este apartado será necesariamente incipiente y limitada, pero es igualmente obvio que las líneas precedentes nos muestran que la lógica formal dispone de bases para explicar fenómenos relativos a silogismos como el que hemos analizado. Por lo demás, es evidente también, como avanzamos más arriba, que la explicación precedente referente a la alteración de los pasos 5, 6 y 7 de la deducción puede aplicarse a cualquier silogismo de la primera figura con independencia del contenido temático que presente.

\section{Conclusiones}

No es nueva la idea de que uno de los problemas de la ciencia cognitiva es que unos mismos resultados experimentales pueden ser interpretados desde distintos marcos teóricos y, por ello, pueden permitir extraer diferentes conclusiones. En este trabajo, hemos podido comprobar que el tema de las conclusiones que se pueden derivar de los silogismos no es ajeno a dicho problema. De esta manera, nuestra intención en estas páginas no ha sido abogar por un enfoque teórico concreto con el propósito de mostrar sus mayores potencialidades o que permite plantear argumentos más sólidos. Nuestra única finalidad ha sido verificar que contamos con marcos alternativos que permiten acercarse a las dificultades que entraña el razonamiento silogístico de diferente manera y adoptando supuestos distintos, los cuales, como se ha podido notar más arriba, pueden no corresponder precisamente a la misma concepción de la cognición humana y llevarnos a asumir diferentes tesis.

En este sentido, creemos oportuno reiterar que, tal y como nos indican Espino y Santamaría, MMT predice sus resultados y estos últimos son claramente consistentes con los planteamientos que se derivan de dicha teoría. Ello es indiscutible. Empero, se puede decir igualmente que la lógica formal también dispone de los recursos necesarios para explicar sus hallazgos y las diferencias que encontraron entre sus condiciones experimentales. 
Por supuesto, lo hemos indicado explícitamente, para ser aceptado definitivamente, un enfoque basado en la lógica formal tendría que responder a algunos interrogantes (en concreto, a los relativos a la traducción de las representaciones mentales al lenguaje de FOPC y a los motivos por los que en unos casos las derivaciones siguen un orden de pasos y en otros un orden diferente). Sin embargo, MMT también va acompañada de sus enigmas, puesto que, como Espino y Santamaría reconocen, tampoco está claro absolutamente por qué en determinadas situaciones los individuos optan por la primera de las estrategias propuestas por MMT y en otras por la segunda estrategia postulada por esta teoría.

Tenemos, de esta manera, una vez más, distintos supuestos y distintas explicaciones que pueden ser, al menos en principio, consistentes con unos mismos resultados experimentales. Es obvio, claro está, que necesitamos mayores investigaciones para poder llegar a saber por cuáles de esos supuestos debemos decantarnos y cuáles debemos descartar. Un indicio en este sentido bien puede ser el alto poder predictivo que, como nos demuestra la literatura, posee MMT en diferentes áreas y ámbitos. No obstante, creemos que, a pesar de ello, no carece de sentido recordar que la lógica también cuenta con posibilidades (aunque estas sean bastante básicas) para ir ofreciendo soluciones a problemas como el examinado en estas páginas.

\section{Nota}

Este artículo es resultado del Proyecto N 1120007 "El procesamiento de enunciados condicionales en los estudiantes de Educación Media: Un estudio a partir de la tarea de selección de Peter Wason", del Fondo Nacional de Desarrollo Científico y Tecnológico (Fondecyt), de la Comisión Nacional de Investigación Científica y Tecnológica (Conicyt), Gobierno de Chile. El autor, que es el investigador responsable del mencionado Proyecto, agradece al programa Fondecyt y al Gobierno de Chile el financiamiento de este trabajo.

\section{Bibliografía}

Espino, O. y Santamaría, C. 2013. Presentation format and syllogistic reasoning. Psicológica 34: 313-326. http://www.uv.es/revispsi/articulos2.13/10Espino.pdf

Johnson-Laird, P. y Steedman, M. 1978. The psychology of syllogisms. Cognitive Psychology 10: 64-99. http://mentalmodels.princeton.edu/papers/1978syllog.pdf

Recibido el 12 Ene 2014

Aceptado el 26 Ago 2014 